\title{
МОДЕЛЬ ОПТИМАЛЬНОГО ЦІНОУТВОРЕННЯ В РЕЖИМІ РЕАЛЬНОГО ЧАСУ НА ОСНОВІ МЕТОДІВ ДИНАМІЧНОГО ПРОГРАМУВАННЯ
}

\section{МОДЕЛЬ ОПТИМАЛЬНОГО ЦЕНООБРАЗОВАНИЯ В РЕЖИМЕ РЕАЛЬНОГО ВРЕМЕНИ НА ОСНОВЕ МЕТОДОВ ДИНАМИЧЕСКОГО ПРОГРАММИРОВАНИЯ}

\author{
OPTIMAL REAL-TIME PRICING MODEL BASED ON DYNAMIC \\ PROGRAMMING METHODS
}

\begin{abstract}
У иій статті проведено аналіз сучасних методологічних підходів до дослідження проблем динамічного иіноутворення, виявлено їх обмеження $і$ критичні питання щзодо практичної реалізації. Більшість робіт передбачає безперервний перегляд цінової політики та обізнаність продавия у функиії попиту, щзо зазвичай важко зустріти на практиці. 3 огляду на це автором запропонована власна модель динамічного прайсингу в дискретному часі з періодичним коригуванням иінової політики. Модель враховує також параметричну («ідеальну») криву продажів $i$ функиію розподілу купівельних иін. Ці характеристики оцінюються за допомогою історичних даних. Завдання моделі - запропонувати оптимальну ціну пропозиції, яка б дозволяла продавати товар згідно темпу параметричної кривої $i$ максимізувала прибуток підприємства. Запропонована методика була успішно протестована для випадку продажу туристичних турів. Подальша розробка моделі передбачає використання байєсівських мереж для калібрування оцінок ї̈ параметрів.
\end{abstract}

Ключові слова: динамічне ціноутворення, управління дохідністю, динамічне програмування, процес Пуассона.

В статье проведен анализ современных методологических подходов к исследованию проблем динамического иенообразования, выявлены их ограничения и критичные вопросы реализации. Большинство подходов предусматривает непрерывный пересмотр ценовой политики и осведомленность продавиза о функиии спроса, что редко предоставляется возможным на практике. В виду этого авторами предложена собственная модель динамического прайсинга в дискретном времени с периодической корректировкой ценовой политики. Модель учитывает также параметрическую («идеальную») кривую продаж $и$ функцию распределения покупательских иен. Эти характеристики оцениваются при помощи исторических данных. Задача модели - выявить оптимальную цену предложения, которая бы позволяла осуществлять продажи согласно темпу параметрической кривой $u$ максимизировала прибыль предприятия. Описанная методика была успешно протестирована для случая продаж туристических туров. Дальнейшая разработка модели предполагает применение обучения байесовских сетей для калибровки оценок её параметров.

Ключевые слова: динамическое ценообразование, управление доходностью, динамическое программирование, процесс Пуассона.

This article analyzes the modern methodological approaches to the study of dynamic pricing problems. It reveals their limitations and critical implementation issues. Most of the working papers suppose the continuous review of pricing policies and the knowledge of the demand function. Such hypothesis is rarely possible in practice. This is why the author suggests his own discrete-time dynamic pricing model with periodic adjustment of the pricing policy. The model also takes into 
account the parametric ("ideal") sale curve and the distribution function of consumer prices. These characteristics are estimated by analysing historical data. The goal of this model is to identify an optimal price. The given price would allow to carry out the sale in accordance to parametric sale curve and to maximize the profit of the firm. This technique has been successfully tested for the case of the package holiday industry. Further development of the model involves the use of Bayesian learning to calibrate the estimation of its parameters.

Keywords: dynamic pricing, revenue management, dynamic programming, Poisson process.

Вступление. Методы динамического ценообразования (или прайсинга) в настоящее время широко используются в таких областях как: розничная и оптовая торговля скоропортящихся товаров, ритейлинг, электронная коммерция, пассажирские перевозки, туризм и т.д. Основная цель предприятий, практикующих динамическое ценообразование, - это максимизация с помощью него конечной прибыли.

Возможность цифровых продаж, которую предоставляет Интернет, позволяет компаниям иметь базу данных, содержащую важную информацию о поведении потребителей, в частности, о том, как они реагируют на различную отпускную цену продукции.

Разумное использование статистических данных, содержащихся в таких базах, и применение методов их преобразования имеет как теоретический интерес, так и неоспоримую практическую актуальность при принятии решений по вопросам формирования ценовой политики. Эти знания могут обеспечить тому или иному предприятию ключевые конкурентные преимущества на рынке.

В основном модели динамического прайсинга базируются на изучении ценообразования услуг или скоропортящихся товаров, так как они имеют следующие характеристики:

1. Количество товара фиксировано и его пополнение затруднительно либо невозможно в рамках одного сезона продажи;

2. Товар или услуга имеет ограниченный срок продажи;

3. Предельная стоимость продажи дополнительной единицы товара (услуги) мала, поэтому большая часть дохода идет непосредственно в прибыль. К примеру, продажа дополнительного пассажирского места влечет малые предельные издержки для авиаперевозчика по сравнению с выручкой, полученной за авиабилет.

Таким образом, этот перечень позволяет также отнести к разряду скоропортящихся товаров посадочные места в самолете и туристические путевки.

В научной литературе, посвященной изучению динамического ценообразования, предполагается, что предприятие имеет некоторое количество единиц продукции, которое должно быть продано в течение конечного периода времени ([1], [2], [3]). При этом пополнение запасов в данный период считается невозможным.

Каждая единица непроданной продукции является безвозвратно потерянной для продавца после окончания горизонта продажи, и не может быть отложена на будущие сезоны продаж. 
В таких задачах, динамический характер оптимальных цен зависит не от изменений в функции спроса, а от изменения предельной стоимости оставшихся запасов с течением времени.

В работе [1] было впервые охарактеризовано и проанализировано стратегию оптимального ценообразования в таком контексте.

Основным и относительно недавним базовым трудом по этой тематике является работа [2]. В ней рассматривается случай, когда спрос моделируется как Пуассоновский процесс в непрерывном времени, характеризующийся коэффициентом интенсивности появления клиентов. Этот коэффициент зависит от цены продажи.

Проблема ценообразования формулируется как задача стохастического оптимального управления, а её оптимальное решение выражается с помощью уравнения Гамильтона-Якоби-Беллмана.

Здесь предложено решение в замкнутой форме только для экспоненциальной функции спроса при соблюдении определенных условий.

Первое: существование взаимно-обратного соответствия между ценой и уровнем спроса. То есть $\lambda(p)$ имеет обратную функцию $p(\lambda)$. Такое представление позволяет получить альтернативный взгляд на установление оптимальной цены. В этом случае предприятие определяет целевой объем спроса $\lambda$, а рынок определяет цену, основанную на этом количестве $p(\lambda)$.

Второе: функция доходности, $r(\lambda)=\lambda p(\lambda)$, должна удовлетворять $\lim _{\lambda \rightarrow 0} r(\lambda)=0$, являться непрерывной, ограниченной, вогнутой и иметь ограниченный максимум, определенный, как $\lambda^{*}=\min \left\{\lambda: r(\lambda)=\max _{\lambda \geq 0} r(\lambda)\right\}$.

Предложенная в работе [2] стратегия оптимального прайсинга предполагает непрерывное изменение цены предложения, что трудно реализовать на практике. Исходя из этого, ученые также разработали два эвристических алгоритма по решению проблемы:

1. алгоритм, основанный на детерминированном варианте задачи;

2. алгоритм, определяющий оптимальную цену, которая остается неизменной на протяжении всего периода продаж.

Эти две эвристики являются асимптотически оптимальными в случае, когда количество предполагаемых продаж достаточно велико, либо когда период продажи стремится к нулю.

Авторы также предлагают другие эвристические подходы и демонстрируют их асимптотическую оптимальность для случаев, когда:

- множество допустимых цен является дискретным, а не непрерывным;

- функция спроса зависит как от цены, так и от времени, прошедшего с начала периода продажи;

- наличие постоянных затрат и ставки дисконтирования;

- целевой переменой модели является начальное количество продукции, а не цена за единицу; 
- случай, когда пополнение запасов, аннулирование покупки или избыточная продажа (овербукинг ${ }^{1}$ ) возможны.

Весомым недостатком их моделей является предположение того, что функция спроса может быть однозначно определена продавцом. Тогда как на практике реализовать это крайне сложно.

В научных статьях по данной тематике можно также найти разнообразные дополнения к изначальной модели Галего и Ван Ризина, в частности:

- модели с ограничениями на множество допустимых цен и на способы их изменения (Фенг и Галего (1995) [3], Битран и Моншейн (1997) [4], Фенг и Сяо $(2000 \mathrm{a}$, б) [5]);

- с функциями спроса, зависящими от времени (Фенг и Галего (2000) [6], Чжао и Чжэнг (2000) [7]);

- приложения для многоканальных продаж (Битран и др. (1998)) [8].

- приложения для нескольких типов одновременно продаваемой продукции (Галего и Ван Ризин (1997) [9], Клейвет (2001) [10]).

Эти разработки базируются на той же гипотезе конечности ресурсов и функции спроса, известной продавцу.

Еще одним важным направлением исследований является изучение стратегического поведения клиентов. В постановке задачи для таких моделей клиент предпочитает выждать некоторое время с целью выиграть на возможном понижении цены, а не принимать решение о покупке немедленно. Тогда в определенный момент времени спрос на продукцию зависит уже не только от актуальной цены продажи, но и от практикуемой в прошлом, и от возможной в будущем.

Одними из примеров исследований в области динамического ценообразования с учетом стратегического поведения клиентов являются работы Левина (2008) [11], Су (2007, 2010) [12], Пазгаль Авива (2008) [13], и др. (2009) [14]. В них наблюдается постановка задач в терминах теории игр, так как обе стороны, предприятие и клиенты, являются стратегическими и имеют конфликтующие интересы.

Постановка задания. В данном исследовании была поставлена задача определения оптимальной цены предложения на текущий момент времени с помощью разработки алгоритма динамического ценообразования и имитационной модели, которая использует статистические данные продаж, учитывает специфические характеристики предприятия и параметрическую («идеальную») кривую продаж. Эта кривая служит сравнительным параметром имитационного эксперимента, и позволяет фирме с помощью динамического установления цен продавать свою продукцию в том темпе, в котором это целесообразно для данного вида товара.

С одной стороны, такая стратегия позволяет избежать дефицита товара в определенный момент до конца срока продажи, а с другой - продавать свой товар тому клиенту, который готов за него платить в данный момент больше, чем остальные.

\footnotetext{
1 Овербукинг (англ. Overbooking) — стратегия сбыта товаров (услуг), при которой поставщик товаров (услуг) принимает на себя больше обязательств по поставке товара (предоставлению услуг), чем может выполнить, в расчете на то, что не все из взятых обязательств действительно придется выполнять.
} 
В целях упрощения хода исследования и интерпретации результатов были использованы некоторые общепринятые в научной литературе по данной тематике гипотезы:

I. Первая и наиболее сильная из них - несовершенная конкуренция (монополия либо олигополия). Под влиянием конкуренции, цена по большему счету регулируется рынком. Если рыночная цена увеличивается, то предприниматель может также увеличить свои цены. С другой стороны, когда рынок в целом снижает уровень цены на данный товар, то предприниматель вынужден также снизить и собственную, чтобы не потерять свою долю рынка.

В условиях отсутствия конкуренции предприятие может установить оптимальную для него цену, которая зависела бы только от флуктуаций спроса. В большинстве исследований по управлению доходностью и, следовательно, ценообразованием авторы не рассматривают рынок. Это происходит в виду того, что данные конкурентов зачастую являются инсайдерской информацией, заполучить которую порой очень трудно, а иногда и просто невозможно.

В нашей модели мы также будем рассматривать предприятие в условиях несовершенной конкуренции. Случай монополии может быть следствием уникальности товара, его новизны, углубленной дифференциации либо применения определенного ноу-хау при технологии производства. В этом случае предприятие-монополист может влиять на спрос лишь варьированием цены. Олигополия, как ещё один вид несовершенной конкуренции, может служить описанием для многих индустрий. Такой рынок состоит из нескольких фирм с большим количеством потребителей. Эти фирмы имеют значительное влияние на соотношение спрос-цена. Примеры олигополий: производство автомобилей, авиаперевозки, телефонные компании.

II. Отсутствие случаев аннулирования совершенной покупки либо овербукинга. В нашей модели не допускается избыточная продажа товара, что обычно практикуется, к примеру, в гостиничном бизнесе либо авиа-перевозках с расчетом на аннулирование брони либо неявку клиента.

III. Предполагается, что появление клиента (то есть брони или заявки на покупку) является случайным процессом Пуассона. Этот процесс, как частный случай марковского процесса, часто используется для описания однородного потока событий, которые могут произойти в любое время с постоянной вероятностью независимо от будущих либо уже произошедших событий.

IV. Каждый клиент знает свою покупательскую цену. Она определяется как максимальная сумма, которую клиент готов заплатить за единицу товара. Таким образом, если цена товара равна $p$, то любой клиент, который готов заплатить $p_{1} \geq p$, купит данный товар.

Методология. Экономико-математический аппарат: методы имитационного моделирования, методы оптимального управления, методы динамического программирования, финансово-экономический анализ, статистические методы обработки временных рядов, стохастические методы, методы эмпирических и теоретических исследований, программные средства современной вычислительной техники (Statistica Advanced Models, Statistica Neural Networks, MS Office Excel, Matlab). 
Результаты исследования. Для построения модели введем условные обозначения.

$C$ - начальный сток; $\lambda$ - средняя интенсивность появления клиентов за единицу времени; [T,0] - конечный горизонт продажи.

Отрицательное значение на шкале времени представляет собой количество дней до конца периода продажи (например, до конца срока годности продукта либо до даты вылета авиарейса). Соответственно, нулевое значение представляет собой последний день, когда возможно реализовать товар либо услугу.

Пусть функция $f(\cdot)$ определена как плотность распределения покупательской цены клиентов,

тогда вероятность покупки по цене равна $p$ :

$$
P(p)=\int_{u=p}^{+\infty} f(u) d u=1-\int_{u=0}^{p} f(u) d u=1-F(p)
$$

где $F(p)$ - функция распределения покупательских цен. Эта вероятность стремится к 0 когда $p \rightarrow+\infty$ и стремится к 1 когда $p \rightarrow 0$.

Рассматривается конечное множество предопределенных продавцом допустимых цен $\mathrm{P}=\left\{p_{1}, p_{2} \ldots, p_{K}\right\}$, где $p_{\min }=p_{1}<p_{2}<\ldots<p_{K}=p_{\max }$. Задача продавца продать изначальный объем товаров в период $[T, 0]$ и максимизировать при этом свою прибыль, варьируя цену предложения.

В отличие от авторов изученных раннее теоретических моделей, где производился непрерывный пересмотр цен, мы разделили период $[T, 0]$ на интервалы (не обязательно равные между собой), называемые «периоды принятия решения».

Таким образом, предприятие может самостоятельно варьировать частоту пересмотра своей ценовой политики, что намного реальнее на практике, чем корректировка цены в непрерывном времени.

Интервалы пронумерованы при помощи индекса $i \in\{0,1,2, \ldots, N\}$ и прогоняются в обратном порядке. При этой нумерации интервал под номером 0 соответствует дедлайну продаж.

Количество прибывших клиентов на протяжении $i$-ого периода является случайной переменной, которая описывается нестационарным процессом Пуассона с интенсивностью $\lambda_{t}, t \in[T, 0]$.

Формулировка предполагает, что в одном периоде принятия решения возможен один и более прибывших клиентов. В таком случае уровень продаж в момент времени $t$ по цене $p$ равняется:

$$
\lambda_{t}\left[1-F_{t}(p)\right], t \in[T, 0] .
$$

Пусть $m_{i}(p)$ представляет собой средний уровень продаж в течение периода $i$ в интервал времени $\left[T_{i}, T_{i-1}\right]$, а $X_{i}(p)-$ количество проданных единиц в этом периоде, тогда:

$$
m_{i}(p)=\int_{T_{i}}^{T_{i-1}} \lambda_{t}\left(1-F_{t}(p)\right) d t
$$




$$
\operatorname{Pr}\left[X_{i}(p)=j\right]=\frac{e^{-m_{i}(p)} m_{i}(p)^{j}}{j !}
$$

где $\operatorname{Pr}\left[X_{i}(p)=j\right]$ - вероятность, что предприятие продаст $j$ единиц товара в период $i$.

Через $U$ обозначим множество ценовых политик $u$, которые представляют собой выбор цен для каждого периода $i$. Элементы этого множества должны удовлетворять условию:

$$
\sum_{i} X_{i}\left(p_{k}\right) \leq C \text { et } p_{k} \in \mathrm{P}
$$

Для отслеживания эффективности продаж вводится параметрическая кривая продаж. Она определяет объем продукции, который следует продать в тот или иной период $i$ для достижения оптимального уровня обслуживания и отсутствия дефицита в течение общего срока продажи.

Применим технику динамического программирования для нахождения оптимального решения данной задачи.

Элементы модели определяются следующим образом:

$i$ - номер периода принятия решения;

$c$ - состояние системы, представляющее собой количество оставшихся запасов в начале каждого периода $i$;

$b_{i}$ - количество единиц, которое следует продать в период $i$, определенное параметрической кривой.

Итак, если система находится в состоянии $c$ и необходимо продать $b_{i}$ единиц продукции, $0<b_{i} \leq c \leq C$, то максимальный ожидаемый доход, который возможно получить, начиная с периода $i$ до конца срока продаж, описывается рекуррентным уравнением (или уравнением динамического программирования):

$$
\begin{gathered}
V_{i}\left(c, b_{i}\right)=\max _{u \in U} \sum_{j=0}^{b_{i}} \operatorname{Pr}\left[X_{i}(p)=j\right] \cdot\left[j p+V_{i-1}(c-j)\right]+ \\
+\operatorname{Pr}\left[X_{i}(p)>b_{i}\right] \cdot\left[b_{i} p+V_{i-1}\left(c-b_{i}\right)\right], \quad i=\overline{0, N}
\end{gathered}
$$

С конечными условиями $V_{i}(0)=0, \forall i$ и $V_{0}(c)=0$ для $c \geq 0$, что означает, что доход невозможен, если запасы либо время продажи уже исчерпаны.

Цель - это найти такие допустимые оптимальные ценовые политики, которые зависят от периода принятия решения $i$ и оставшегося стока $c$ : $p=p_{i}(c)$. Поиск решения для этого уравнения осуществляется с помощью индукции «назад во времени» (обратный прогон).

Пусть цена предложения пересматривается каждую неделю, тогда этапы поиска решения следующие:

1. Определение множества допустимых цен.

2. Расчет, то есть максимального кол-ва единиц, которое фирма планирует продать в период.

3. Построение матрицы ожидаемых доходностей (Табл.1.): 
Матрица ожидаемых доходностей

\begin{tabular}{|c|c|c|c|c|c|c|}
\hline $\begin{array}{c}\text { Дни до } \\
\text { истечения } \\
\text { срока } \\
\text { продажи }\end{array}$ & $i$ & 0 & 1 & 2 & $\ldots$ & $\mathrm{C}$ \\
\hline 0 & 0 & $\mathbf{0}$ & $\mathbf{0}$ & 0 & $\mathbf{0}$ & 0 \\
\hline$[0,-7]$ & 1 & $\mathbf{0}$ & $V_{1}\left(1, b_{1}\right)$ & $V_{1}\left(2, b_{1}\right)$ & $\ldots$ & $V_{1}\left(C, b_{1}\right)$ \\
\hline$[-7,-14]$ & 2 & $\mathbf{0}$ & $V_{2}\left(1, b_{2}\right)$ & $V_{2}\left(2, b_{2}\right)$ & $\ldots$ & $V_{2}\left(C, b_{2}\right)$ \\
\hline$\ldots$ & $\cdots$ & $\cdots$ & $\ldots$ & $\cdots$ & $\cdots$ & $\cdots$ \\
\hline$[-231,-238]$ & $\mathrm{N}$ & $\mathbf{0}$ & $V_{N}\left(1, b_{N}\right)$ & $V_{N}\left(2, b_{N}\right)$ & $\ldots$ & $V_{N}\left(C, b_{N}\right)$ \\
\hline
\end{tabular}

4. Находясь в начале периода $\mathrm{N}$, производим обратный расчет во времени. Разрешая сперва задачу максимизации ожидаемого дохода нулевого периода, поэтапно восходим к задаче максимизации ожидаемых доходов предшествующих нулевому периодов вплоть до текущего периода $\mathrm{N}$ (Табл.2.):

Таблица 2

Матрица обратного прогона цен

\begin{tabular}{|c|c|c|c|c|c|c|}
\hline $\begin{array}{c}\text { Дни до } \\
\text { истечения } \\
\text { прока } \\
\text { продажи }\end{array}$ & $i$ & 0 & 1 & 2 & $\ldots$ & C \\
\hline 0 & 0 & $\mathbf{0}$ & $\mathbf{0}$ & $\mathbf{0}$ & $\mathbf{0}$ & $\mathbf{0}$ \\
\hline$[0,-7]$ & 1 & $\mathbf{0}$ & $p_{1}(1)$ & $p_{1}(2)$ & $\ldots$ & $p_{1}(C)$ \\
\hline$[-7,-14]$ & 2 & $\mathbf{0}$ & $p_{2}(1)$ & $p_{2}(2)$ & $\ldots$ & $p_{2}(C)$ \\
\hline$\ldots$ & $\ldots$ & $\ldots$ & $\ldots$ & $\ldots$ & $\ldots$ & $\ldots$ \\
\hline$[-231,-238]$ & $\mathrm{N}$ & $\mathbf{0}$ & $p_{N}(1)$ & $p_{N}(2)$ & $\ldots$ & $p_{N}(C)$ \\
\hline
\end{tabular}

Величина ожидаемого дохода в периоде 0 дана конечным условием $V_{0}(c)=0$.

Таким образом, задача максимизации величины $V_{1}\left(c, b_{1}\right)$ может быть легко получена при переборе всех возможных цен.

После нахождения цены, которая максимизирует правую часть уравнения, мы ее сохраняем в соответствующей матрице оптимальных цен. И переходим к задаче максимизации величины $V_{2}\left(c, b_{1}\right)$ и т.д.

Построение параметрической кривой. Для функционального представления параметрической кривой мы использовали бета-распределение, так как оно позволяет охватить множество других распределений за счет 
вариации параметров. Плотность вероятности бета-распределения определяется, как:

$$
\begin{aligned}
f(x ; \alpha, \beta) & =\frac{x^{\alpha-1}(1-x)^{\beta-1}}{B(\alpha, \beta)} \\
\text { где } B(\alpha, \beta) & =\int_{0}^{1} x^{\alpha-1}(1-x)^{\beta-1} d x
\end{aligned}
$$

Оценка параметров бета-распределения по историческим данным продаж осуществлялась при помощи метода моментов. Если через $\mu$ и $\sigma^{2}$ обозначить математическое ожидание и дисперсию соответственно, то оценки параметров распределения могут быть получены из формул:

$$
\begin{gathered}
\hat{\alpha}=\mu\left(\frac{\mu(1-\mu)}{\sigma^{2}}-1\right) \\
\hat{\beta}=(1-\mu)\left(\frac{\mu(1-\mu)}{\sigma^{2}}-1\right)
\end{gathered}
$$

Оценка функции распределения покупательских цен. При оценке функции распределения покупательских цен использовался метод, применяемый Ю. Левиным и др. в работе «Риск при управлении доходностью» [11]. Сначала из статистических данных оценивается параметр $\lambda_{t}$, который соответствует количеству прибывших клиентов в момент времени $t$. Для представления этих данных в функциональной форме использовалось приложение «Curve fitting tool», предоставляемое MatLab. Теперь если через $\bar{\lambda}_{t}(p)$ обозначить количество клиентов, которые совершили покупку в момент времени $t$ по цене $p$, то величину $\bar{\lambda}_{t}(p) / \lambda_{t}$ можно интерпретировать как вероятность, что клиент, прибывший согласно пуассоновскому процессу с интенсивностью $\lambda_{t}$ покупает товар по цене $p$.

Следовательно, для каждого периода $i$ и для каждой допустимой цены функцию распределения вероятности в форме $F_{i}(p)=1-\bar{\lambda}_{t}(p) / \lambda_{t}, t \in\left[T_{i+1}, T_{i}\right]$ можно рассматривать как функцию распределения покупательских цен.

Bсе необходимые расчеты производились с помощью встроенных функций Excel, Statictica и Matlab.

Разработанная нами модель в дискретном времени с конечным множеством допустимых цен и параметрической кривой продаж была успешно протестирована для случая продажи пакетных туристических туров (перелет+трансфер+отель).

Статистические данные были предоставлены туристической компанией «Promovacances» для даты вылета 17/07/2015, направление Катания (Сицилия). Начальный сток составлял $C=71$ туристических тура, которые были открыты к продаже за 240 дней до даты вылета.

В качестве начальной (базовой) цены была выбрана цена, установленная в начале периода продажи экспертами отдела «Управление доходностью». Интервал между ценами мы установили на уровне 5\% по отношению к базовой цене, а максимальное повышение (понижение) цены на уровне $+30 \%(-30 \%)$.

Таким образом, зафиксировав начальную цену на уровне 822 евро, мы получили следующее множество допустимых цен: 


$$
\mathrm{P}=\{575,616,657 \ldots, 1069\}
$$

Оцененные параметры бета-распределения позволили построить следующую параметрическую кривую для данной даты вылета (рис. 1).

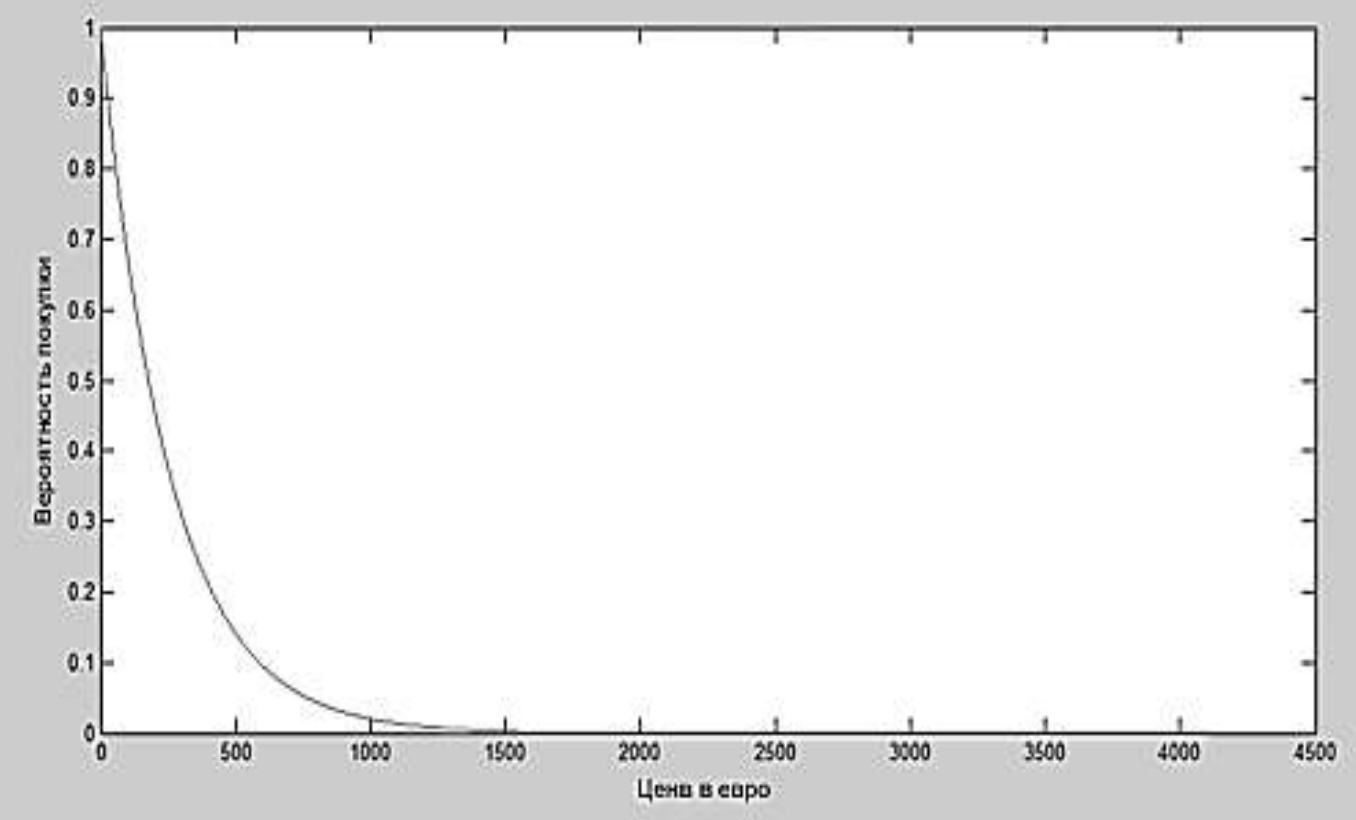

Рис.1. Параметрическая кривая продаж

Оценивая функцию распределения покупательских цен согласно методу, описанному выше и сглаживая полученную кривую, мы смогли представить её в экспоненциальной форме $F(p)=1-e^{-\alpha p}, p>0$, где $\alpha=256$.

Таким образом, вероятность покупки, то есть $1-F(p)$, представлена на следующем графике (рис.2):

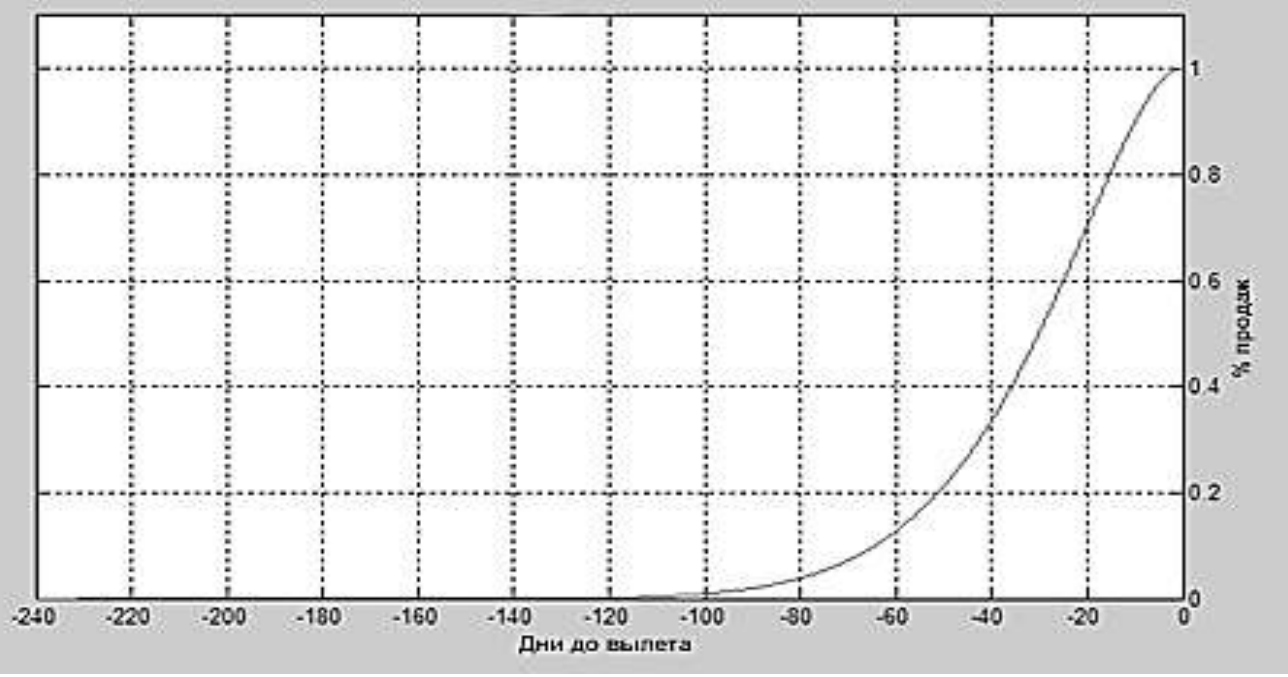

Рис. 2. Вероятность покупки (цена в евро)

На рис.3 представлена траектория оптимальных ценовых индексов, начиная с 240 дней до даты вылета вплоть до последнего дня продажи туров с пересмотром ценовой политики каждые 7 дней. Ценовой индекс рассчитывается делением текущей цены на базовую цену. Такой индекс дает понимание осуществляемых корректировок цены: индекс больший, чем 
единица, указывает на повышение цены по сравнению с изначально фиксированной, а индекс меньший единицы, соответственно, на понижение.

Тонкая ступенчатая сплошная кривая симулирует процент продаж в начале каждого периода, пунктирная ступенчатая кривая представляет собой рассчитанные с помощью параметрической кривой $b_{i}$ для соответствующего периода.

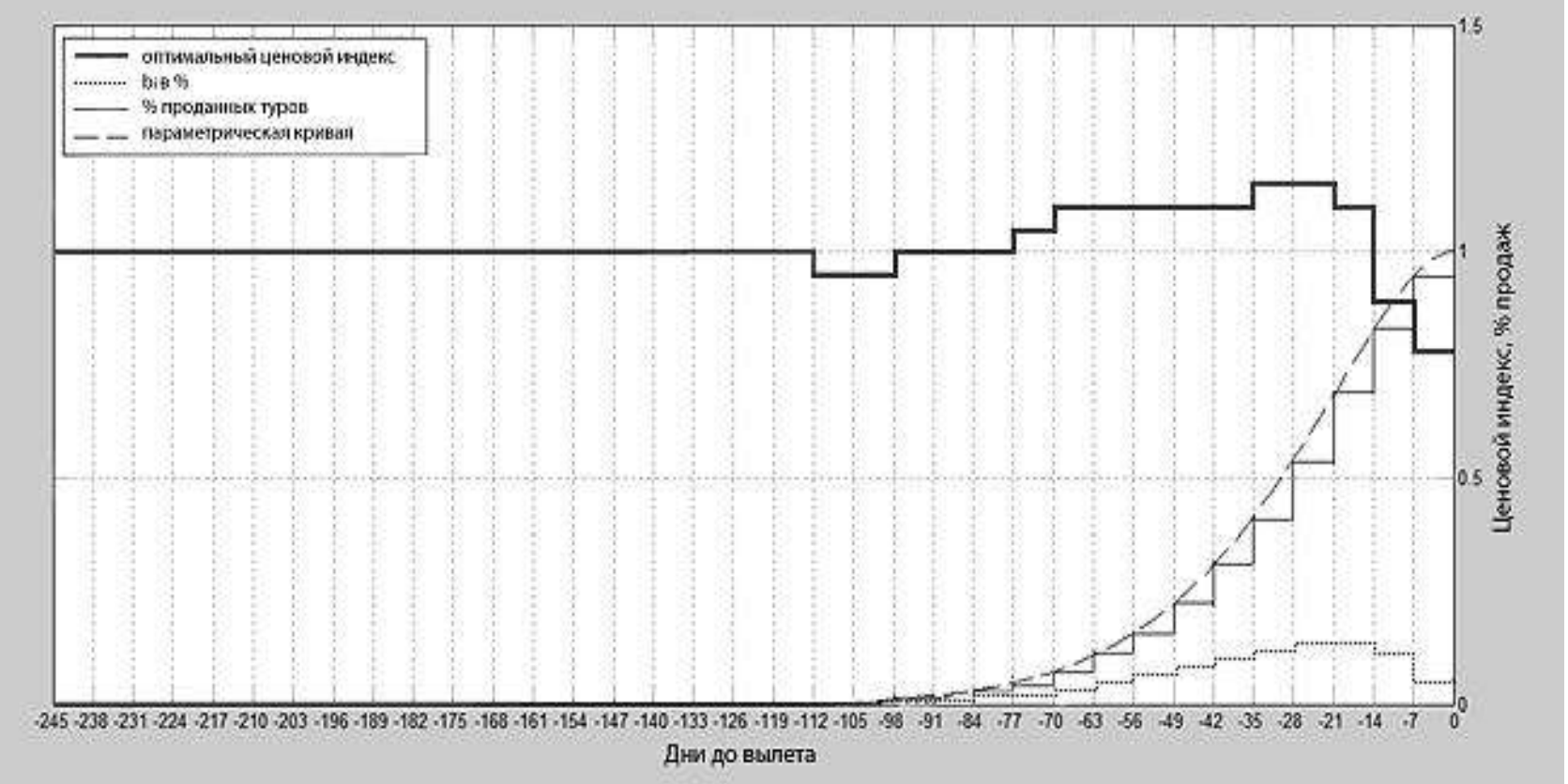

Рис. 3. Траектория оптимальных ценовых индексов

Вплоть до 112 дня до вылета модель сохраняет изначально зафиксированную цену (жирная кривая), так как согласно оцененной параметрической кривой в этот период практически не наблюдается продаж. Как можно заметить, несмотря на то, что $b_{i}$ возрастают в период между 35 и 21 днями до вылета, модель предлагает повысить цену предложения.

Это происходит за счет того, что интенсивность прибытия клиентов в этот период наиболее высока. В этом случае, повышая цену, туроператор продает путевки только тем клиентам, которые готовы за них платить больше всего. Тем не менее, к концу горизонта продаж туроператор вынужден снизить свои цены, чтобы не оказаться с пустыми местами в самолете.

Выводы. Научная новизна статьи состоит в предложенном алгоритме динамического ценообразования, на базе которого реализована имитационная модель. Она позволяет на основе статистических данных продаж, определять оптимальную цену предложения на текущий момент, учитывая при этом специфические характеристики предприятия и параметрическую («идеальную») кривую продаж. Эта кривая служит сравнительным параметром имитационного эксперимента, и позволяет фирме с помощью динамического установления цен продавать свою продукцию в том темпе, в котором это целесообразно для данного вида товара. С одной стороны такая стратегия позволяет избежать дефицита товара в определенный момент до конца срока продажи, а с другой продавать свой товар тому клиенту. В отличие от моделей в непрерывном 
времени, построенная модель предлагает пересмотр ценовой политики с той частотой, которая целесообразна для определенного вида товара.

Практическое значение разработанной модели состоит в том, что учет параметрической кривой продаж позволяет контролировать интенсивность реализации товара, стимулируя или тормозя спрос за счет варьирования цены.

Исследование модели позволило также теоретически подтвердить тот факт, что оптимальная цена возрастает с убыванием запасов при фиксированном времени и убывает при приближении концу срока продажи при фиксированном уровне запасов. Следует также отметить, что чем обширнее база статистических данных продаж, тем выше точность результатов, предоставляемых данной моделью. Задача предприятия правильно организовать сбор и классификацию таких данных, что повысит точность оценки функции распределения покупательских цен и параметрической кривой продаж.

В перспективе научного исследования - создание самообучающейся модели, которая бы использовала как исторические данные, так и данные о текущих продажах, и калибровала оценки необходимых параметров. Теоретической основной такого подхода может послужить байесовское обучение с заданием априорного распределения вероятностей.

\section{Литература:}

1. Kincaid W.M., D. Darling. 1963. An inventory pricing problem, Journal of Mathematical Analysis and Applications 7 (1963) 183-208.

2. Gallego, G., G. van Ryzin. 1994. Optimal dynamic pricing of inventories with stochastic demand over finite horizons. Management Sci. 40(8)999-1020.

3. Feng, Y., G. Gallego. 1995. Optimal starting times for end-of-season sales and optimal stopping times for promotional fares. Management Science, 41(8):1371-1391.

4. Bitran, G.R., S.V. Mondschein. 1997. Periodic pricing of seasonal products in retailing. Management Sci.

5. Feng, Y., B. Xiao. 2000a. À continuous time yield management model with multiple prices and reversible price changes. Management Sci. 46(5) 644-657.

6. Feng, Y., B. Xiao. 2000b. Optimal policies of yield management with multiple predetermined prices. Oper. Res. 48(2) 332-343.

7. Feng, Y., G. Gallego.2000. Perishable asset revenue management with Markovian time dependent demand intensities. Management Science, 46(7):941-956.

8. Zhao, W., Y. Zheng. 2000. Optimal dynamic pricing for perishable assets with non homogeneous demand. Management Sci., 46(3): 375-388.

9. Gallego, G., G. van Ryzin. 1997. A multiproduct dynamic pricing problem and its applications to network yield management. Oper. Res. 45(1) 24-41.

10. Kleywegt, A. J. 2001. An optimal control problem of dynamic pricing. Working paper. Management Sci. 41(1) 221-236.

11. Levin, Y., McGill, J., M. Nediak. 2008. Risk in revenue management and dynamic pricing, Operations Research 56 (2008) 326-343.

12.X. Su. Intertemporal pricing with strategic customer behavior. Management Science, 53(5):726-741, 2007.

13. Aviv, Y., \& Pazgal, A. (2008). Optimal Pricing of Seasonal Products in the Presence of Forward-Looking Cnsumers. Manufacturing and Service Operations Management, 10 (3), 339-359.

14. Levin, Y., J. McGill, M. Nediak. 2009. Dynamic pricing in the presence of strategic consumers and oligopolistic competition. Management Sci. 55(1) 32-46. 